\title{
Quantitative D-dimer level and anticoagulant therapy in idiopathic intracranial hypertension
}

\author{
Hala Shaheen, Sayed Sobhy, Sherine El Mously * D, Mohamed El Khatib and Amany Hamdy
}

\begin{abstract}
Background: Idiopathic intracranial hypertension $(I I H)$ is a syndrome characterized by elevated intracranial pressure (ICP) of unknown etiology

Aim of the work: The aim of this research is to study the quantitative D-dimer level and the role of anticoagulant therapy in absence of occlusive sinus thrombosis in patients with $\mathrm{IH}$.

Methodology: Twenty-four patients with $\mathrm{IH}$ according to the modified Dandy criteria were enrolled. Headache impact test (HIT6), ophthalmological assessment including Frisen classification for papilledema, visual acuity, visual field, and visual evoked potentials were performed to the patients. Serum quantitative D-dimer level was measured using the enzyme-linked immunosorbent assay (ELISA) technique for the patients and for 24 healthy matched controls. Patients were divided into two groups: group (1) received acetazolamide and low molecular weight heparin (LMWH) in a prophylactic dose for 2 weeks while group (2) received acetazolamide only. Both groups continued on acetazolamide for 6 months. We followed the patients after 1 and 6 months later through the HIT6 test and the ophthalmological assessment.
\end{abstract}

Results: D-dimer level was statistically higher among the cases compared to the controls. Also, a statistically significant improvement was recorded in the ophthalmological assessment after 6 months among both groups; more evident in group (1).

Conclusion: The elevated D-dimer level and the visual improvement in IIH patients receiving LMWH added to acetazolamide suggest the presence of an underlying unrecognized non-occlusive venous cerebral microthrombi impeding the cerebrospinal fluid (CSF) drainage.

Trial registration: ClinicalTrials,gov on 22/5/2019, NCT03963336.

Keywords: Idiopathic intracranial hypertension, D-dimer, Microthrombi, Anticoagulant therapy

\section{Introduction}

Idiopathic intracranial hypertension (IIH) is a syndrome characterized by elevated intracranial pressure (ICP) in the absence of intracranial disorder, a meningeal process or cerebral venous thrombosis [1]. The common clinical manifestations are headache and visual dysfunction in the form of blurring of vision, field defects, and binocular diplopia [2].

The pathophysiology underlying the raised ICP still remains obscure but there are some proposed mechanisms

\footnotetext{
*Correspondence: smm02@fayoum.edu.eg

Faculty of Medicine, Fayoum University, Fayoum 63611, Egypt
}

such as increased brain water content, excessive cerebrospinal fluid (CSF) production or reduced absorption, and increased cerebral venous pressure. It was noticed that obese young women and patients having bilateral transverse sinus stenosis are predisposed to develop increased ICP. Endocrinal changes, hypercoagulable state, or specific medications may trigger the increased venous pressure [3].

Moreover, a prothrombotic state with an elevated Ddimer level has been observed in obese IIH patient [4]. The D-dimer is considered the smallest fibrinolysis-specific degradation product in the circulation and it is an exquisite sensitive test to intravascular thrombosis [5]. 
The aim of our study was to measure the serum quantitative D-dimer level in IIH patients and to investigate the role of anticoagulation added to the routine treatment as a possible effective therapy.

\section{Patients and methods}

This is a prospective case control study. We recruited 24 consecutive IIH patients of both sexes fulfilling the modified Dandy criteria [6] from the Neurology department, Fayoum University Hospitals in the period from January to October 2017.

We excluded patients with disturbed conscious level, focal neurological signs, seizures, cerebral venous sinus thrombosis or stenosis, deep venous thrombosis or pulmonary embolism, malignancy, and acute nephritic syndrome. Patients with signs of disseminated intravascular coagulopathy and septic sinus thrombosis and patients with history of cerebral granuloma or infection, cerebrovascular stroke, head trauma, or surgery were excluded. Finally, pregnant females or those who terminated their pregnancy in the last 4 weeks were also excluded.

The study population was divided into two groups randomly; patients with odd number were included in group (1) while those with even number were fitted in group (2). Group (1) received acetazolamide and low molecular weight heparin (LMWH) in a prophylactic dose $1 \mathrm{mg} / \mathrm{kg} /$ day for 2 weeks, while group (2) received acetazolamide only. Both groups continued on acetazolamide (1-2 g/ day) for 6 months.

Twenty-four age, sex, and body mass index (BMI) matched healthy volunteers were selected as a control group. They were the patients' relatives visiting the Neurology clinic.

All patients were subjected to thorough history taking and full neurological examination. We assessed the headache severity using the headache impact test (HIT6) that is a six-item test used to provide a global measure of the adverse headache impact. The final HIT-6 score is obtained from simple summation of the six items and ranges between 36 and 78, with lower scores reflecting lower impact [7]. There is no Arabic validated translated version for the HIT-6 test up till now; we translated it and we referred to the score present in the original one. Patients were evaluated for headache severity at baseline then after 1 and 6 months.

The ophthalmological assessment included a fundus examination with grading of papilledema according to Frisen classification [8]. Also, visual acuity was assessed using the Snellen's chart. The results obtained were converted into the logarithm of minimum angle of resolution (log MAR) for analysis [9]. Finally, visual field assessment was performed by the automated perimetry (SBP, 3000S) that measures the visual field mean deviation (MD). An MD of 0 to $2 \mathrm{~dB}$ indicates a normal value, while a negative value represents a deviation [10]. All patients were subjected to these ophthalmological tests at baseline and at follow up after 1 and 6 months in the Ophthalmology Department.

Visual evoked potentials (VEPs) using the Nicolet Viking IV equipment was performed. The latency and the amplitude of P100 wave was measured. Responses were considered abnormal if the P100 latency exceeded $110.85 \mathrm{~ms}$ in any eye or if the response amplitude was absent [11].

Serum quantitative D-dimer level (normal level up to $200 \mathrm{ng} / \mathrm{dl}$ ) was assessed using the enzyme-linked immunosorbent assays (ELISA) technique. Venous blood samples $(2 \mathrm{~cm})$ were obtained from the patients and the controls to be centrifuged immediately. The separated plasma was stored at $2-8{ }^{\circ} \mathrm{C}$. The ELISA Kit is based on the principle of competitive binding. Patient samples, standards, and controls were acidified and neutralized before starting the procedure's steps. The micro titer wells were coated with a monoclonal antibody directed toward the D-dimer molecule. The pre-treated sample was incubated at room temperature with the conjugate biotinylated D-dimer. The wells were washed then incubated with the enzyme complex streptavidin-horse radish peroxidase (HRP). After adding the substrate solution, the concentration of the D-dimer was calculated in a reverse proportional manner to the intensity of the color developed.

\section{Ethical consideration}

The study was approved by the Research Ethical Committee of the Faculty of Medicine, Fayoum University. All the participants either patients or controls signed an informed and detailed consents after explaining for them the aim and the procedures of the study. The confidentiality of their data and their right not to participate in the study were respected.

\section{Statistical analysis}

Statistical package for social sciences (SPSS) version 18 for Microsoft Windows was used for data management and analysis. The sample size was calculated according to Epi Info 2000. A sample size of 24 was calculated using a special formula at a confidence interval of $95 \%$ and precision of $(2 \%)$. Simple descriptive analysis in the form of numbers and percentages was performed for qualitative data and arithmetic means such as central tendency measurement. Standard deviation (SD) was used as a measure of dispersion for quantitative parametric data and inferential statistic test. For quantitative parametric data, independent student $t$ test was used to compare measures of two independent groups while paired $t$ test was used to compare between two dependent groups. For qualitative data, we applied Chisquare test to compare two of more than two qualitative groups and general linear model to compare repeated 
measures. A $p$ value $\leq 0.05$ was considered statistically significant.

\section{Results}

The present study included $24 \mathrm{IIH}$ patients and 24 controls. Their demographic data are shown in Table 1.

There was neither statistically significant difference between cases and controls nor between IIH groups regarding the age, sex, and BMI $(p>0.05)$.

The D-dimer serum level was high in all IIH patients ranging from 100 to $310 \mathrm{ng} / \mathrm{dl}$ (mean $232.4 \pm 54.5$ ) while in controls it ranged from 100 to $250 \mathrm{ng} / \mathrm{dl}$ (mean 186.1 $\pm 22.7)$. There was a statistically significant difference between the two groups $(p<0.001)$. Meanwhile, group (1) had a mean of D-dimer level $233.4 \mathrm{ng} / \mathrm{dl}$ and group (2) had a mean of $231.4 \mathrm{ng} / \mathrm{dl}$ with no statistically significant difference between the two groups.

The HIT6 score improved in all the patients on follow up as shown in Table 2. Statistically significant differences were found in HIT6 score after 1 and 6 months follow up with low mean in group (2) as shown in Table 3.

Regarding the ophthalmological assessment, $58.3 \%$ of the patients presented with papilledema grade 2 at baseline and they improved gradually on follow up reaching grade $1(41.7 \%)$ at 6 months. The log-MAR was $0.30-$ 0.60 in $41.7 \%$ of the patients at baseline and improved at 6 months follow up reaching $00.00-0.30$ in $66.7 \%$ of the patients. Also, $85.4 \%$ presented with mild to moderate field defect at baseline while at 6 months follow up 83.3\% had mild to normal field defect.

No significant differences were found between IIH groups at baseline visual assessment. A statistically significant difference was found with improvement in papilledema's grade between the baseline assessment and after 6 months between IIH groups; $p$ value in group (1) was less than 0.001 while in group (2) the $p$ value was 0.009 . Similarly, a statistically significant difference was found in log-MAR between the baseline assessment and 6 months follow up; in group (1) $(p=0.003)$ and group (2) ( $p$ value $=0.02)$.

A statistically significant improvement was found in visual field between the baseline assessment and 6 months follow up in group (1) $(p=0.009)$, while there was no statistically significant difference in visual field

Table 1 Demographic data of $॥ \mathrm{H}$ patients and controls

\begin{tabular}{|c|c|c|}
\hline & $\begin{array}{l}\text { IIH Patients } \\
(N=24)\end{array}$ & $\begin{array}{l}\text { Controls } \\
(N=24)\end{array}$ \\
\hline $\begin{array}{l}\text { Age in years } \\
\text { (mean } \pm \text { SD) }\end{array}$ & $\begin{array}{l}16-44 \\
(29.04 \pm 6.6)\end{array}$ & $\begin{array}{l}\text { 17-46 years } \\
(29.1 \pm 6.6)\end{array}$ \\
\hline $\begin{array}{l}\text { Number of } \\
\text { females (\%) }\end{array}$ & $19(79.2 \%)$ & 20 (83.3\%) \\
\hline $\begin{array}{l}\mathrm{BMl} \text { in } \mathrm{kg} / \mathrm{m}^{2} \\
\text { (mean } \pm \mathrm{SD} \text { ) }\end{array}$ & $\begin{array}{l}24-61.22 \\
(36.3 \pm 8.3)\end{array}$ & $\begin{array}{l}19.5-55 \\
(34.8 \pm 8.1)\end{array}$ \\
\hline
\end{tabular}

SD standard deviation, $B M I$ basal metabolic index
Table 2 HIT6 score at baseline, after 1 and 6 months

\begin{tabular}{llll}
\hline HIT 6 score & $\begin{array}{l}\text { Baseline } \\
(n=24)\end{array}$ & $\begin{array}{l}\text { After 1 month } \\
(n=24)\end{array}$ & $\begin{array}{l}\text { After 6 months } \\
(n=24)\end{array}$ \\
\hline Little or no impact (<49) & - & 11 & 14 \\
Some impact (50-55) & 4 & 7 & 10 \\
Substantial impact (56-59) & 10 & 4 & - \\
Severe impact (60-78) & 10 & 2 & - \\
\hline
\end{tabular}

HIT6 headache impact test 6

between the baseline and 6 months follow up in group (2) $(p=0.2)$. Also, no statistically significant difference was found between both groups from the baseline to 6 months follow up ( $p$ value was 0.8 and 0.4 respectively). A statistically significant decline in VEP latencies and a statistically significant increase in VEP amplitudes were found between the baseline assessment and 6 months follow up between both IIH groups as shown in Fig. 1 and (Table 4).

\section{Discussion}

The IIH is a syndrome of elevated ICP with obscure etiology. An underlying prothrombotic state would explain at least a part of its pathogenesis [12]. The D-dimer is a by-product of fibrinolysis and has an already established role in the diagnosis of venous thromboembolism and disseminated intravascular coagulation [13].

Our results showed a statistically significant higher mean of D-dimer among the cases compared to the controls. This result agrees with Kesler A. et al. 2010 [4]

Headache is the most common presenting symptom in IIH [2]. The HIT6 score improved statistically in all the patients on follow up after 1 and 6 months which agrees with Friedman D. and his colleagues who used the acetazolamide as a treatment for IIH [14].

Statistically significant differences were found in HIT6 score after 1 and 6 months follow up with lower mean in group [2] indicating that the headache improved much more in patients who did not receive anticoagulant therapy. This discrepancy between both groups in

Table 3 Comparisons of HIT6 score at baseline, after 1 and 6 months follow up between $I \mathrm{H}$ groups

\begin{tabular}{llll}
\hline HIT6 & $\begin{array}{l}\text { Group 1 }(n=12) \\
\text { Mean } \pm \text { SD }\end{array}$ & $\begin{array}{l}\text { Group 2 }(n=12) \\
\text { Mean } \pm \text { SD }\end{array}$ & $p$ value $^{a}$ \\
\hline Baseline & $58.58 \pm 3.17$ & $58.58 \pm 3.17$ & 1 \\
1 month & $55.8 \pm 3.1$ & $45 \pm 2.6$ & $<0.001$ \\
6 months & $51.1 \pm 2.2$ & $41.1 \pm 2.2$ & $<0.001$ \\
$p$ value $^{b}$ & 0.003 & 0.002 & \\
\hline
\end{tabular}

HIT6 headache impact test 6, SD: standard deviation

${ }^{a}$ Significance between both groups

${ }^{b}$ Significance in each study group 


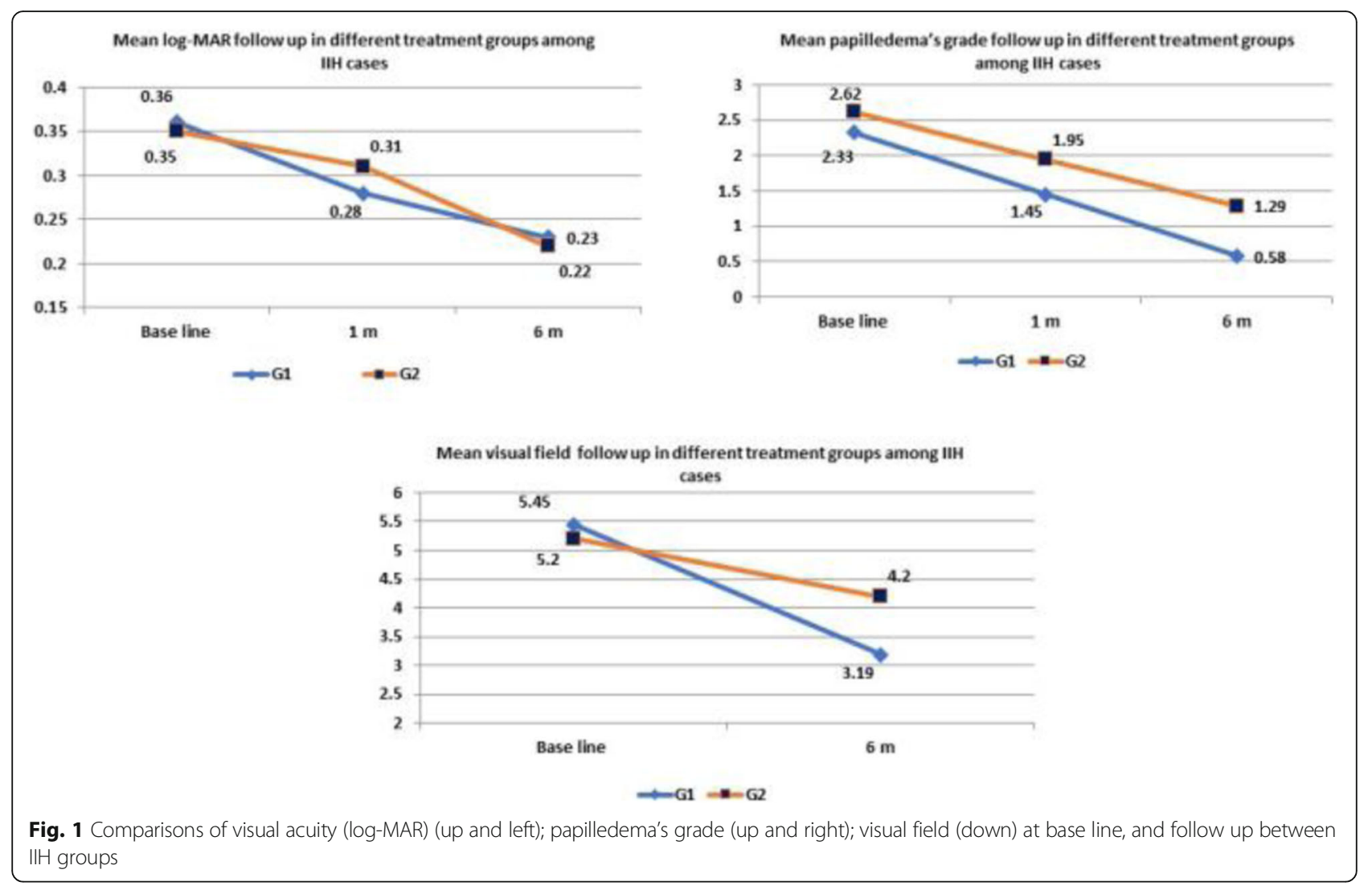

headache improvement can be explained by the fact that the present headache could be associated with tension type headache, depression, or psychosomatic symptoms. Nevertheless, the HIT6 score is a subjective evaluating method and not objective.

Regarding the ophthalmological assessment, our results showed a statistically significant improvement in papilledema's grade at 6 months follow up among both

Table 4 Comparisons of VEP latency and amplitude at baseline and after 6 months follow up between $1 \mathrm{H}$ groups

\begin{tabular}{llll}
\hline $\begin{array}{l}\text { VEP } \\
\text { latency }\end{array}$ & $\begin{array}{l}\text { Group (1) }(n=12) \\
\text { Mean } \pm \text { SD }\end{array}$ & $\begin{array}{l}\text { Group [2] }(n=12) \\
\text { Mean } \pm \text { SD }\end{array}$ & $p$ value $^{a}$ \\
\hline Latency & & $100.9 \pm 11.3$ & 0.8 \\
Base line & $101.9 \pm 4.2$ & $95.8 \pm 5.6$ & 0.02 \\
6 months & $87.3 \pm 9.9$ & $<0.001$ & \\
$p$ value $^{b}$ & $<0.001$ & & \\
Amplitude $^{\text {Base line }}$ & $11.5 \pm 5.4$ & $10.6 \pm 3.4$ & 0.6 \\
6 months & $12.2 \pm 5.4$ & $11.2 \pm 3.5$ & 0.6 \\
$p$ value $^{b}$ & 0.02 & 0.006 & \\
\hline
\end{tabular}

VEP visual evoked potentials

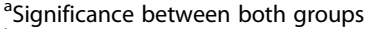

${ }^{\mathrm{b}}$ Significance in each study group groups which agrees with Wall $M$ [15] who explained that the acetazolamide works by inhibition of carbonic anhydrase leading to reduction in sodium ions transport across the choroid plexus epithelium. There was a statistically significant decrease in papilledema after 6 months among patients who received anticoagulant treatment with acetazolamide while no statistically significant difference between both treatment groups after 1 month may be because the papilledema takes time to resolve.

Concerning the visual acuity, there was a statistically significant improvement in log-MAR after 6 months among both groups of patients which agrees with Wall M 15]. The improvement was more noticed in patients who received anticoagulant therapy.

Visual field assessment was considered the main criterion to assess the clinical improvement in IIH patients [16]. There was a statistically significant improvement in visual field after 6 months among patients who received anticoagulant therapy and acetazolamide. On the other hand, there was no difference in visual field follow up among patients who did not receive anticoagulation which disagrees with Wall M. [15] who found an improvement in visual field after 6 months among IIH patients who received acetazolamide added to low sodium diet. Moreover, Celebisoy N. and co-authors [16] showed visual field improvement on either acetazolamide or topiramate 
considering that the two medications exert their effect through the carbonic anhydrase inhibition. Nevertheless, Koc F and his colleagues 2018 [17] showed in their recent study that the weight reduction combined with acetazolamide significantly improve the visual field in IIH patients. The discrepancy in the results could be attributed to different methodology applied in the studies.

Finally, the VEPs results showed a statistically significant decrease in latency and significant increase in amplitude after 6 months among both groups with more improvement among the group who received anticoagulant therapy. This result was in accordance with Hamurcu M. and co-authors [18] who reported that the papilledema initially does not disrupt the function of the optic nerve and retinal nerve fiber layer (RNFL), but it may lead to an elongation of the p100 latency in VEP test and an increase in RNFL. After elimination of the effect of edema, changes in the optic nerve and RNFL improve. When papilledema is not treated and becomes chronic, ischemic symptoms may occur with the effect of pressure on the nerve fibers and RNFL. Therefore, early diagnosis and treatment are extremely important.

Up to our knowledge, this study is the first to investigate the quantitative D-dimer serum level in IIH patients and to study the role of anticoagulant therapy in IIH cases. The results obtained prove the possibility of an unrecognized non-occlusive venous cerebral thrombus impeding the CSF drainage as described in previous studies [3, 4]. We can assume that the anticoagulant therapy disrupted these microthrombi and improved the CSF drainage which was manifested by the improvement of the papilledema, visual field, visual acuity, and the VEPs results.

The limitations of this study were the small sample size and the absence of objective instrumental findings demonstrating the presence of unrecognized non-occlusive venous cerebral thrombus impeding the CSF drainage.

To conclude, our study demonstrated the presence of high serum quantitative D-dimer levels among the IIH patients and showed an improvement in visual assessment after 6 months in both groups of patients; more apparent in the cohort who received anticoagulant therapy added to the acetazolamide. Routine screening of D-dimer level is recommended in patients with $\mathrm{IIH}$. Further studies are warranted for objective instrumental findings able to detect this unrecognized non-occlusive venous cerebral thrombus. Also, larger studies are needed to establish guidelines for the use of anticoagulation in IIH patients.

\footnotetext{
Abbreviations

BMI: Body mass index; CSF: Cerebrospinal fluid; ELISA: Enzyme-linked immunosorbent assay; HIT6: Headache impact test; HRP: Horse radish peroxidase; ICP: Intracranial pressure; IIH: Idiopathic intracranial hypertension; LMWH: Low molecular weight heparin; log MAR: Logarithm of minimum angle of resolution; MD: Mean deviation; RNFL: Retinal nerve fiber layer; SBP: Standard base program; SD: Standard deviation; SPSS: Statistical package for social sciences
}

\section{Acknowledgements}

We acknowledge all the staff members of the Ophthalmology Department for their great help in the ophthalmological assessment of the cases. We acknowledge also all the staff members in Clinical Pathology department who handled the laboratory part of the work. Finally, special thanks to Dr. Asmaa Younes, lecturer of Community for her help in the statistical analysis.

\section{Authors' contribution \\ $\mathrm{AH}$ recruited the cases and collected all the needed data. SEM, SS, and HS revised the clinical data obtained and the results. Finally, MEK wrote the manuscript which was revised by the other authors to be ready for publication. SEM is the corresponding author who is responsible for the publication. All authors read and approved the final manuscript.}

\section{Funding}

No funding was obtained from any institution for our study.

\section{Availability of data and materials}

Data can be available for publication only by special approval from the Fayoum University.

\section{Ethics approval and consent to participate}

The study was approved by the Faculty of Medicine Research Ethical Committee of Fayoum University in its $33^{\text {rd }}$ session on the $11^{\text {th }}$ of January 2017, number M201. A written consent was taken from all the participants either cases or controls after being informed about the objectives of the study, the examination and the investigations. The confidentiality of their information was respected and their right not to participate in the study was ensured.

\section{Consent for publication}

A consent for publication was obtained from all the participants included in the study. The manuscript does not contain any individual person's data.

\section{Competing interests}

The authors declare that they have no competing interests.

Received: 28 April 2019 Accepted: 19 August 2019

Published online: 05 September 2019

\section{References}

1. Friedman DI, Jacobson DM. Diagnostic criteria for idiopathic intracranial hypertension. Neurology. 2002;59:1492-5.

2. Smith SV, Friedman DI. The idiopathic intracranial hypertension treatment trial: a review of the outcomes. Headache. 2017;(8):1303-10.

3. Biousse V, Bruce BB, Newman NJ. Update on the pathophysiology and management of idiopathic intracranial hypertension. J Neurol Neurosurg Psychiatry. 2012;83:488-94.

4. Kesler A, Kliper E, Assayag EB, Zwang E, Deutsch V, Martinowitz U, Lubetsky A, Berliner S. Thrombophilic factors in idiopathic intracranial hypertension; a report of 51 patients and a meta-analysis. Blood Coagul Fibrinolysis. 2010; 21(4):328-33.

5. Olson JD. D-dimer: an overview of hemostasis and fibrinolysis, assays, and clinical applications. Adv Clin Chem. 2015;69:1-46.

6. Friedman DI, Liu GT, Digre KB. Revised diagnostic criteria for the pseudotumor cerebri syndrome in adults and children. Neurology. 2013;81: 1159-65.

7. Kosinski M, Bayliss MS, Bjorner JB, Ware JE Jr, Garber WH, Batenhorst A, Cady R, Dahlöf CG, Dowson A, Tepper S. A six-item short-form survey for measuring headache impact: the HIT-6. Qual Life Res. 2003;12(8):963-74.

8. Sinclair AJ, Burdon MA, Nightingale PG, Matthews TD, Jacks A, Lawden M, Sivaguru A, Gaskin BJ, Rauz S, Clarke CE, Ball AK. Rating papilloedema: an evaluation of the Frisén classification in idiopathic intracranial hypertension. J Neurol. 2012;259(7):1406-12.

9. Ma Y, Huang J, Zhu B, Sun Q, Miao Y, Zou H. Cost-utility analyses of cataract surgery in advanced age-related macular degeneration. Optom Vis Sci. 2016;93(2):165-72.

10. Nouri-Mahdavi K. Selecting visual field tests and assessing visual field deterioration in glaucoma. Can J Ophthalmol. 2014 Dec;49(6):497-505.

11. Walsh P, Kane N, Butler S. The clinical role of evoked potentials. J Neurol Neurosurg Psychiatry. 2005;76(2):ii16-22. 
12. De Lucia D, Napolitano M, DiMicco P,Niglio A, Fontanella A, and Di lorio G Benign intracranial hypertension associated to blood coagulation derangements. Thromb J. 2006; 4: 21.

13. Soomro AY, Guerchicoff A, Nichols DJ Suleman J, Dangas GD. The current role and future prospects of D-dimer biomarker. European Heart Journal—Cardiovascular Pharmacotherapy. 2016;(2):3; 175-3; 184.

14. Friedman DI, Quiros PA, Subramanian PS, Mejico L, Gao S, McDermott M, Wall $M$. Headache in idiopathic intracranial hypertension: findings from the idiopathic intracranial hypertension treatment trial. Headache. 2017;57:1195-205.

15. Wall M. Effect of acetazolamide on visual Function in patients with idiopathic intracranial hypertension and mild visual loss; the idiopathic intracranial hypertension treatment trial. AMA. 2014;311(16):1641-51.

16. Celebisoy N, Gökçay F, Sirin H, Akyürekli O. Treatment of idiopathic intracranial hypertension: topiramate vs acetazolamide, an open-label study. Acta Neurol Scand. 2007 Nov:116(5):322-7.

17. Koc F, Isik MR, Sefi-Yurdakul N. Weight reduction for a better visual outcome in idiopathic intracranial hypertension. Arq Bras Oftalmol. 2018;81(1):18-23.

18. Hamurcu M, Sarıcaoğlu M, Koca S, Ekicier S, Karakurt A. Visual evoked potential and optical coherence tomography in pseudotumor cerebri. Research. 2015;2:1476.

\section{Publisher's Note}

Springer Nature remains neutral with regard to jurisdictional claims in published maps and institutional affiliations.

\section{Submit your manuscript to a SpringerOpen ${ }^{\circ}$ journal and benefit from:}

- Convenient online submission

- Rigorous peer review

- Open access: articles freely available online

High visibility within the field

Retaining the copyright to your article 